\title{
Learning Statistics from Counter Examples: Ancillary Statistics
}

\author{
D. Basu ${ }^{1}$
}

\begin{abstract}
Bayesian objection to the analysis of data in frequency theory terms is amplified through several connter examples in which an ancillary statistic exists and there is a temptation to choose a reference set after looking at the data. It is argued that Fisher insisted on conditioning by an ancillary statistic, because conditioning the data $\mathbf{x}$ by an ancillary $Y$ does not change the likelihood. In this sense Fisher discovered the supremacy of the likelihood function.
\end{abstract}

Key words and Phrases: Ancillary statistics; Conditional frequentist inference; Information; Liklihood principle; Reference set; Sufficiency principle.

\section{INTRODUCTION}

This paper is especially addressed to the statisticians who have not yet fully grasped the Bayesian objection to the analysis of data in repeated sampling terms. Let $\mathbf{x}$ be the sample, $f(\mathbf{x} \mid \theta)$ the model and $\theta$ the parameter. A statistic $Y=Y(\mathbf{x})$ is ancillary if the sampling distribution of $Y$, given $\theta$, is $\theta$-free (is the same for all values of $\theta$ ). A statistic $T=T(\mathbf{x})$ is sufficient if the distribution of the sample $\mathbf{x}$, given $T$ and $\theta$, is $\theta$-free. An ancillary statistic $Y$ by itself contains no information about the parameter, whereas a sufficient statistic $T$ is fully informative in a sense. R.A. Fisher's attempt to make sense of the notion of information in the data led him to these two important concepts in Statistics.

Let $L(\theta)=f(\mathbf{x} \mid \theta)$ be the likelihood function determined by the sample $\mathbf{x}$ and let $\hat{\theta}$ be the maximum likelihood (ML) estimate of $\theta$. If $\hat{\theta}$ is a sufficient statistic then, according to Fisher, there would be no loss of information if the performance characteristics of $\hat{\theta}$ as an estimate of $\theta$ is sought to be evaluated in terms of the sampling distribution of $\hat{\theta}$. We shall repudiate this in the end with an example.

\footnotetext{
${ }^{1}$ Indian Statistical Institute and Florida State University
} 
If the ML estimate $\hat{\theta}$ is not a sufficient statistic then Fisher sought to recover the information lost in the sampling distribution of $\hat{\theta}$ with the help of an ancillary complement $Y$ to the estimator $\hat{\theta}$. The ancillary statistic $Y$ has to complement $\hat{\theta}$ in the sense that the pair $(\hat{\theta}, Y)$ is jointly sufficient. The Fisher Information $I_{\hat{\theta}, Y}(\theta)$ in the sufficient statistic $(\hat{\theta}, Y)$ is then the same as the full information

$$
I(\theta)=-E\left[\frac{\partial^{2}}{\partial \theta^{2}} \log L(\theta)\right]
$$

in the sample $\mathbf{x}$. (Note that $I(\theta)$ does not relate to the particular sample $\mathbf{x}$ but is obtained by averaging the quantity $-\frac{\partial^{2}}{\partial \theta^{2}} \log L(\theta)$ over the sample space.) The Fisher Information in the statistic $\hat{\theta}$ is less than the full information $I(\theta)$. The cornerstone of the Fisher argument lies in the identity

$$
I(\theta)=I_{\hat{\theta}, Y}(\theta)=E\left[I_{\hat{\theta}}(\theta \mid Y)\right],
$$

where $I_{\hat{\theta}}(\theta \mid Y)$ is the conditional information in the statistic $\hat{\theta}$, given $Y$, and the expectation on the right hand side is with respect to the ancillary statistic $Y$. Thus, the conditional information in $\hat{\theta}$, given $Y$, depends on $Y$ and can be, for a particular value of the statistic $Y$, much less or much greater than the full information $I(\theta)$. The conditionality argument of R.A. Fisher rests on the proposition that the performance characteristics of the estimator $\hat{\theta}$ ought to be evaluated conditionally, holding the ancillary statistic $Y$ fixed at its observed value $y$. As Fisher argued, the event $Y=y$, even though uninformative by itself, has a lot of latent information about $\theta$ in the sense that it helps us discern how good or bad the estimate $\hat{\theta}$ is in the present instance. The set $S(y)=\{\mathbf{x}: Y(\mathbf{x})=y\}$ defines what Fisher called the reference set. Sir Ronald was trying to cut down the sample space $S$ to size. We illustrate the conditionality argument with several examples.

\section{EXAMPLES}

Example 1: Let $\mathbf{x}=\left(x_{1}, x_{2}, \ldots, x_{n}\right)$ be iid observations on a random variable that is uniformly distributed over the interval $[\theta, 2 \theta]$, where $\theta>0$ is the unknown scale parameter. With

$$
m=\min x_{i} \text { and } M=\max x_{i},
$$

the likelihood function $L(\theta)$ equals $1 / \theta^{n}$ over the interval $[M / 2, m]$ and zero outside the interval. The ML estimator $\hat{\theta}=M / 2$ is not sufficient, the minimal sufficient statistic being the pair $(m, M)$. Since the two statistics $m$ and $M$ are stochastically independent in an asymptomatic sense, it is clear that there will be a substantial loss of information if we marginalize the data to the $\mathrm{ML}$ estimator $M / 2$. Comparing the mean squared error (MSE) of $M / 2$ with that of $m$ as estimators of $\theta$, we find that the former is exactly four times better than the latter. Consider, therefore, the estimator 
$T=(2 M+m) / 5$ which is the weighted average of $M / 2$ and $m$ with weights 4 and 1 respectively. Both $M / 2$ and $T$ are equivariant estimators of the scale parameter $\theta$, and so their MSE's are constant multiples of $\theta^{2}$. It works out that the ratio of the two MSE's tends to 25/12 as the sample size $n$ tends to infinity. The ML estimator $\hat{\theta}$ can hardly be called an efficient estimate of $\theta$ in the usual sense of the term. Over thirty-six years ago, when I came upon this counterexample, it was pointed out to me by C.R. Rao that the ML estimator $\hat{\theta}$ ought to be judged conditionally after holding fixed its ancillary complement $Y=M / m$ at its observed value. That $Y$ is an ancillary statistic follows from the facts that $Y$ is scale invariant and that $\theta$ is a scale parameter. As we noted before, the likelihood mass is spread over the interval $[M / 2, m]$ pinpointing the parameter $\theta$ within that interval. The statistic $Y=M / m$ varies over the range $[1,2]$ and is indeed a measure of how good the sample is - the nearer $Y$ is to 2 the better the sample is. While evaluating the ML estimate $\hat{\theta}$ we ought to take note of the observed value $y$ of the statistic $Y$. That is, instead of referring $\hat{\theta}$ to the full sample space $S$, we ought to refer it to the reference set $S(y)$.

In terms of the full sample space $S$ the ML estimator $M / 2$ is not sufficient. But when it is conditioned by $Y$ it suddenly becomes fully informative (sufficient, that is). Note that the other two estimators $m$ and $T$ also become fully informative when they are referred to the set $S(y)$. Indeed, the three statistics $M / 2, m$ and $T$ become functionally related when conditioned by $Y$.

This example beautifully illustrates what Fisher meant by recovery of ancillary information. The next example illustrates how a weak pivotal quantity can be strengthened by proper conditioning with an ancillary statistic.

Example 2: Let $\mathbf{x}=\left(x_{1}, x_{2}, \ldots, x_{n}\right)$ be $n$ iid observations on a random variable with pdf $f(x-\theta)$, where $f$ is known but $\theta$ (the location parameter) is unknown. Consider the statistic $x_{1}$ and its ancillary complement $D=$ $\left(x_{2}-x_{1}, x_{3},-x_{1}, \ldots, x_{n}-x_{1}\right)$. The statistic $x_{1}$ by itself carries very little information about $\theta$, but it becomes fully informative (sufficient) when conditioned by $D$. The conditional pdf of $x_{1}$, given $D$, has $\theta$ embedded in it as a location parameter. Fisher derived the fiducial distribution of the parameter $\theta$ by inverting the pivotal quantity $x_{1}-\theta$ after conditioning it by the ancillary statistic $D$.

The previous example raises many questions. Some sample questions and answers are listed below.

Question: What is the status of the ancillary statistic $D$ ? Is it the maximum ancillary in the sense that every other ancillary statistic is a function of $D$ ?

Answer: No. $D$ is never the maximum ancillary. However, in some situations $D$ will be a maximal ancillary in the sense that no larger (with respect to the partial order of functional relationship) ancillary statistic exists. A 
multiplicity of maximal ancillaries is a fact of life in this situation.

Question: Is the fiducial distribution of $\theta$ in Example 2 critically dependent on the choice of the pivotal quantity $x_{1}-\theta$ ?

Answer: No. Another pivotal quantity like, say, $\mathbf{x}-\theta$, when conditioned by $D$, will result in the same fiducial distribution of 0 . This is because $\bar{x}=x_{1}+\left(\bar{x}-x_{1}\right)$ and $\bar{x}-x_{1}$ is a function of $D$.

Question: Can we interpret the fiducial distribution of $\theta$ probabilistically?

Answer: It was pointed out by Harold Jeffreys that the fiducial distribution of the location parameter (as derived by Fisher) coincides with the posterior distribution of $\theta$ corresponding to the uniform prior (over the entire real line) for the parameter.

In the presence of multiple ancillaries, the choice of the proper reference set is a problem. The dilemma is best exemplified by the following example.

Example 3: Let $\left(x_{1}, y_{1}\right), i=1,2, \ldots, n$, be $n$ iid observations on $(X, Y)$ whose joint distribution is Bivariate Normal with zero means, unit variances and covariance $\theta$, which is the parameter of interest. In this case both $\mathbf{x}=\left(x_{1}, \ldots, x_{n}\right)$ and $\mathbf{y}=\left(y_{1}, \ldots, y_{n}\right)$ are ancillary statistics. Note that the pair $(\mathbf{x}, \mathbf{y})$ is the entire data and therefore is sufficient. Holding the ancillary $\mathbf{x}$ as fixed and regarding $\mathbf{y}$ as the variable, we may want to estimate $\theta$ by $\sum x_{i} y_{i} / \sum x_{i}^{2}$ and then regard the estimate as unbiased with variance $(1-$ $\left.\theta^{2}\right) / \sum x_{1}^{2}$. But how about holding $\mathbf{y}$ fixed and reporting that $\sum x_{i} y_{i} / \sum y_{i}^{2}$ is an unbiased estimate with variance $\left(1-\theta^{2}\right) / \sum y_{1}^{2}$ ? It is tempting to opt for the ancillary with the larger sum of squares. But would it not be a statistical heresy to choose the reference set after looking at the data?!

\section{COX ON ANCILLARIES}

D.R. Cox (1971) suggested a way to deal with the problem of multiple ancillaries. Looking back at the Fisher identity $I(\theta)=E I(\theta \mid Y)$, Cox argued that the basic role of the conditioning ancillary $Y$ is to discriminate between samples with varying degrees of information. So in the presence of multiple ancillaries we should choose that $Y$ for which $I(\theta \mid Y)$ is most variable in $Y$. So opt for the $Y$ for which $\operatorname{Var} I(\theta \mid Y)$ is maximum. One snag in the Cox argument is that $\operatorname{Var} I(\theta \mid Y)$ is a function of $\theta$ and so there may not exist a $Y$ that maximizes the function uniformly in $\theta$. Also note that in our Example 3 the Cox method fails because, in view of the perfect symmetry between $\mathbf{x}$ and $\mathbf{y}$, $\operatorname{Var} I(\theta \mid \mathbf{x})=\operatorname{Var} I(\theta \mid \mathbf{y})$.

But the real snag in the Cox argument is the meaninglessness of the notion of Fisher Information as a measure of the evidential meaning of the particular data at hand. Fisher's preoccupation with the elusive notion of information in the data led him to the likelihood function which he recognized as the carrier of all the information in the data. The likelihood was then partially summarized in the two statistics $\hat{\theta}$, the ML estimate, and $Z(\hat{\theta})$, the second derivative of $-\log L(\theta)$ at $\theta=\hat{\theta}$. Note that $Z(\hat{\theta})$ is the reciprocal of the radius of curvature of the log likelihood at its mode, the 
larger the value of $Z(\hat{\theta})$ the sharper is the fall of the likelihood function as $\theta$ moves away from $\hat{\theta}$. We have to stretch our minds a little to regard $Z(\hat{\theta})$ as a rough measure of the concentration of the likelihood mass around $\hat{\theta}$. The greater the concentration the more informative is the likelihood. The Fisher Information $I(\theta)$ is obtained from $Z(\hat{\theta})$ by first replacing $\theta$ by $\theta$ and then taking the average value of $Z(\theta)$ over the whole sample space $S$. But how can we regard $I(\theta)$ as information in the data?

Why did Fisher insist that the conditioning statistic $Y$ has to be ancillary? Because, conditoning the data $\mathbf{x}$ by an ancillary $Y$ does not change the likelihood. Fisher discovered the supremacy of the likelihood but got carried away by his amazing craftmanship with sample space mathematics.

\section{E.L. LEHMANN ON ANCILLARIES}

Eric Lehmann (1981) finally recognized the conditionality argument. And now he has to cope with the disturbing presence of ancillary statistics. Invoking the Sufficiency Principle, Eric would reduce the data $\mathbf{x}$ to the minimal sufficient statistic $T=T(\mathbf{x})$. Since $T$ is sufficient, all reasonable inference procedures ought to depend on $\mathbf{x}$ only through $T(\mathbf{x})$. This data reduction sweeps away much of the ancillary dust under the rug. But, as in Example 1, some functions of the minimal sufficient statistic $T$ may still be recognized as ancillary statistics. Eric has yet to come out openly on the question of how to deal with such persistent ancillaries.

From what Eric writes in his 1981 article, it seems that he feels quite comfortable with statistical models for which the minimal sufficient statistic $T$ is complete. In such cases no nontrivial function of $T$ can be ancillary. Furthermore, thanks to the so called Basu Theorem, every ancillary statistic $Y$ is stochastically independent (conditionally on $\theta$ ) of $T$. Therefore, no $T$ - based decision procedure can be altered by conditioning with an ancillary $Y$. So who needs to think of the conditionality argument when we have a complete sufficient statistic? Remember, Fisher looked for an ancillary complement to the ML estimate $\hat{\theta}$ only when the statistic $\hat{\theta}$ was not sufficient. So in the most favorable set up where $\hat{\theta}$ is a complete sufficient statistic, can anyone object if we evaluate the estimate $\hat{\theta}$ in terms of the sampling distribution of the estimator? We give an example to prove both Fisher and Lehmann wrong on this question.

Example 4: Consider a sequence of Bernoulli trials with parameter $p$ that results in a finite sequence $w=S F F S \ldots F S$ of successes $S$ and failures $F$. Let $X(w)$ and $Y(w)$ denote, respectively, the number of $S$ 's and the number of $F$ 's in the sample sequence $w$. We picture $w$ as a sample path, the locus of a point that begins its journey at the original and travels through the lattice points of the positive quadrant, moving one step to the right for each $S$ and one step up for each $F$. The lattice point with coordinates $X(w)$ and $Y(w)$ is where the sample path $w$ ends. Our example relates to a particular 
sampling (stopping) rule $\mathbf{R}$. Writing $(X, Y)$ for the location of the moving point, the rule is described as:

Rule R: Continue sampling as long as (I) $Y<2 X+1$, (II) $Y>X-2$, and (III) $X+Y<100$. Alternatively, the rule may be defined as: Stop sampling as soon as the sample path hits one of the three boundary lines (i) $y=2 x+1$, (ii) $y=x-2$, and (iii) $x+y=100$.

As always, the likelihood does not recognize the stopping rule and comes out as

$$
L(p)=f(w \mid p)=p^{X(w)} q^{Y(w)}
$$

where $q=1-p$. The pair $X(w), Y(w)$ constitute the minimal sufficient statistic. The ML estimate is $\hat{p}=X /(X+Y)$. The range of the sufficient statistic $(X, Y)$ consists of the boundary points

$$
\begin{array}{llllll}
(0,1), & (1,3), & \ldots, & (33,67) & \text { on line } & (i), \\
(34,66), & (35,65), & \ldots, & (50,50) & \text { on line } & (\text { iii }), \text { and } \\
(51,49), & (50,48), & \ldots, & (2,0) & \text { on line } & (\text { ii }) .
\end{array}
$$

The ML estimator $\hat{p}=X /(X+Y)$ monotonically increases from zero to unity as $(X, Y)$ moves through the above set of boundary points. Hence $\hat{p}$ itself is minimal sufficient. Let us assert here without proof that $\hat{p}$ is a complete sufficient statistic in this case and that no nontrivial ancillary statistic exists.

Sir Ronald is no longer with us. So let me address the following questions to my good friend Eric Lehmann who is a living legend among us for his unparalleled erudition in Statistical Mathematics. The questions relate to Example 4.

Question: What should be our criterion for the choice of an estimate of $p$ ?

(The unbiasedness criterion is sort of vacuous in this case. There is only one unbiased estimator, which is zero or unity depending on whether the first trial results in an $F$ or an $S$.)

Question: If ML is the chosen criterion, then how should we evaluate the estimate $\hat{p}=X /(X+Y)$ ? Does it make sense to evaluate $\hat{p}$ in terms of some average performance characteristics?

Question: Are all sample paths $w$ equally informative?

(Even though there are no ancillary statistics in this case, we can still detect major qualitative differences between different sample paths. For instance, short sample paths like $F$ or $S S$ have very little to say about the parameter, whereas long paths that end on line (iii) are clearly much more informative.)

Question: Why do we need to decipher what the sample $w$ has to say about the parameter $p$ in terms of a sample space? Does the sample $F$ 
obtained following the rule $\mathbf{R}$ say anything different from the statement: $\mathbf{A}$ single Bernoulli trial has resulted in a failure?

Question: Do sample space ideas like bias, variance, risk function, etc., make any sense in this case?

Question: Why not act like a Bayesian and analyze the particular likelihood function generated by the data? Isn't is quite clear in this case that all that the data has to say about the parameter is summarized in the likelihood?

\section{REFERENCES}

Basu, D. (1988), Statistical Information and Likelihood: A Collection of Critical Essays by D. Basu, ed. J.K. Ghosh, Springer Verlag, New York.

Cox, D. R. (1971), The Choice Between Alternative Ancillary Statistics. Jour. Royal Statist. Soc. (B)33, 251-255.

Lehmann, E.L. (1981), An Interpretation of Completeness and Basu's Theorem. Jour. Amer. Stat. Assoc., 76, 335-340. 